\title{
Predicting the impact of measles vaccination in England and Wales: model validation and analysis of policy options
}

\author{
H. R. BABAD ${ }^{1}$, D. J. NOKES ${ }^{1 *}$, N. J. GAY ${ }^{2}$, E. MILLER ${ }^{2}$, \\ P. MORGAN-CAPNER ${ }^{3}$ AND R. M. ANDERSON ${ }^{1}$ \\ ${ }^{1}$ Centre for the Epidemiology of Infectious Disease, Department of Zoology, \\ University of Oxford, South Parks Road, Oxford OX1 3PS \\ ${ }^{2}$ Immunisation Division, PHLS Communicable Disease Surveillance Centre, \\ 61 Colindale Avenue, London NW9 5EQ \\ ${ }^{3}$ Public Health Laboratory, Royal Preston Hospital, Sharoe Green Lane North, \\ Preston PR2 4HG
}

(Accepted 30 October 1994)

\section{SUMMARY}

Measles incidence in England and Wales has fallen to an all-time low. Attention is now focused on preventing local outbreaks, and, in the long run, on the elimination of indigenous measles. A realistic age-structured (RAS) mathematical model of measles transmission is used to reconstruct the impact of measles vaccination in England and Wales from 1968 to the present and to evaluate the merits of future policy options. In general, the predictions of the model show good agreement with long-term age stratified case reports and seroprevalence surveys. The model underestimates the proportion of cases that are notified in 0-2-year-old children. However, recent work suggests a high degree of misdiagnosis in this age group. Projections on the basis of the existing vaccination strategy in the UK suggest that the present level of measles vaccine coverage will be insufficient to eliminate small seasonal outbreaks of measles. This result is, however, sensitive to the assumed level of vaccine efficacy. Explorations of a variety of changes to current vaccination strategy favour a 2-dose schedule with the second dose administered at age 4 years irrespective of vaccination history. A vaccination campaign in school-age children, to reduce deficits in herd immunity, would accelerate progress towards measles elimination.

\section{INTRODUCTION}

Since the introduction of measles, mumps, rubella (MMR) triple vaccine in late 1988 [1], the incidence of measles in England and Wales has declined to an all-time low [2], and the characteristic seasonal and 2-yearly epidemic cycles have all but disappeared [3]. However, measles persists, raising the issue of whether the present single dose immunization policy will eventually achieve its intended goal 
of elimination [1] or whether calls for policy change, spurred on by outbreaks in older children, may be justified $[2,4]$. In this paper a mathematical model of measles transmission is described and its predictions verified against a variety of epidemiological observations. Given the good agreement between observation and prediction the model is used to explore measles epidemiology in the future, under the existing vaccination strategy and a variety of alterations to this strategy.

Much is known about the behaviour of measles in England and Wales prior to mass vaccination largely through long-term age-stratified notification records $[3,5]$ and a good understanding of the typical course of infection in the individual and the route of transmission of the virus [6]. Analyses of measles notification time series have yielded useful insights into the major factors responsible for the characteristic biennial epidemicity and seasonal fluctuations prior to mass vaccination [7-11], and these patterns can be well mimicked using deterministic compartmental mathematical models [9-11]. The introduction of mass childhood cohort vaccination drastically alters the stable endemic pattern of infection, and mathematical models facilitate an assessment of the likely impact of a particular vaccination strategy on the epidemiology of measles in a defined population $[10,12]$.

Before such quantitative methods can gain credibility as useful predictive tools there is need to carefully verify predictions with observed epidemiological pattern under the impact of mass vaccination. There is now a growing body of literature which lends support to the accuracy (both quantitative and qualitative) of model predictions $[12,13]$. Specifically, for measles, a set of age-stratified notification data has accumulated since the introduction of national vaccination in 1968 by which to validate model predictions. Unfortunately, notification data is of questionable reliability due to, for example, age-specific bias in reporting [14], and alteration in the efficiency of reporting following the introduction of mass vaccination and the proportion of cases misdiagnosed [15].

An additional measure by which to gauge the ability of mathematical models to capture the epidemiological impact of mass vaccination is through comparison with age-stratified antibody prevalence data. Seroepidemiological studies provide an accurate profile of passive, naturally acquired and vaccine-induced immunity in a population. Age-stratified antibody prevalence data for a range of vaccine preventable diseases is now routinely collected by the Public Health Laboratory Service (PHLS) in England and Wales [16]. These data have been used to assess the impact of mass vaccination on the prevalence of susceptibles, the deficits in measles herd immunity in specific age groups which may be of concern (Gay et al, unpublished) and in parameter estimation, e.g. the age-specific force of infection [17].

In this paper we employ a form of mathematical model previously shown to describe most closely the patterns of measles infection in England and Wales prior to vaccination $[9,11]$. We show to what degree this model is able to capture the characteristics of measles infection at various times after the introduction of mass vaccination. For this we use age-stratified measles notification data, and a series of serological surveys for England and Wales, dating from 1969, 1979-83, 1986 and 1991. Finally, the model is used to evaluate the merits of the current and future policy options for mass vaccination. 


\section{METHODS}

\section{Mathematical model and its construction}

We use a realistic age-structured (RAS) model (a term coined by Bolker and Grenfell [11]) which is based upon the method of Schenzle [9]. This type of model was chosen in preference to previous age-structured models $[10,18]$ because of improved numerical accuracy and the incorporation of a more realistic age- and time-dependent structure [11]. The following provides an overview of the major characteristics of the model. For a formal exposition see the work of Schenzle [9] and Bolker and Grenfell [11].

Two components of this deterministic compartmental model, not present in other age-structured models (see, for example, Anderson and May, 1985 [10]), are important in capturing more precisely the pattern of observed measles incidence prior to mass vaccination. First, a seasonality component representing changes in contact rates which occur due to school-term and vacation patterns. This seasonal input or forcing is implemented by the sequential switching between a high and a low rate of mixing, within primary school-age children (aged 5-9 years), in association with school term and holiday times for England and Wales (and weekday and weekends), respectively.

Second, a cohort age-structure is established by placing individuals, as they are born (between September of one year and September of the next), within yearly birth cohorts. These cohorts subsequently age as discrete units through the age profile. This captures the way children move from one school year to the next as they progress through the education system in a more realistic way than that based on the absolute age of individuals.

To illustrate how the seasonality and cohort structure together may provide a more accurate representation of the spread of measles infection, consider children moving from primary school into secondary school. It is not their absolute age which determines the timing of the move, rather the start of the new school year. Children do not trickle into the higher school as they pass some age gateway, instead, in September the whole of the eligible school year cohort moves into secondary school. This entails a movement of some 600000 children in England and Wales, in the space of a week, coming together in the close confines of school classrooms, experiencing high rates of mixing, with the potential to precipitate a seasonal epidemic which in turn influences the longer-term cycles in incidence (i.e. 2 year cycles).

A further modification of the model which further facilitates a comparison between observed and predicted seroprevalence profiles is a category of individuals who have received vaccine but who are not effectively protected from infection.

\section{Compartmental structure of model}

The diagram shown in Fig. 1 summarizes the model's compartmental structure, and flow of individuals through the various infection and immunity classes. All individuals are assumed to be born protected from measles by specific antibodies passed transplacentally from the mother (class M). This assumes that all mothers have specific antibody acquired either from infection or vaccination. This passive protection wanes over the first year of life leaving individuals susceptible to 


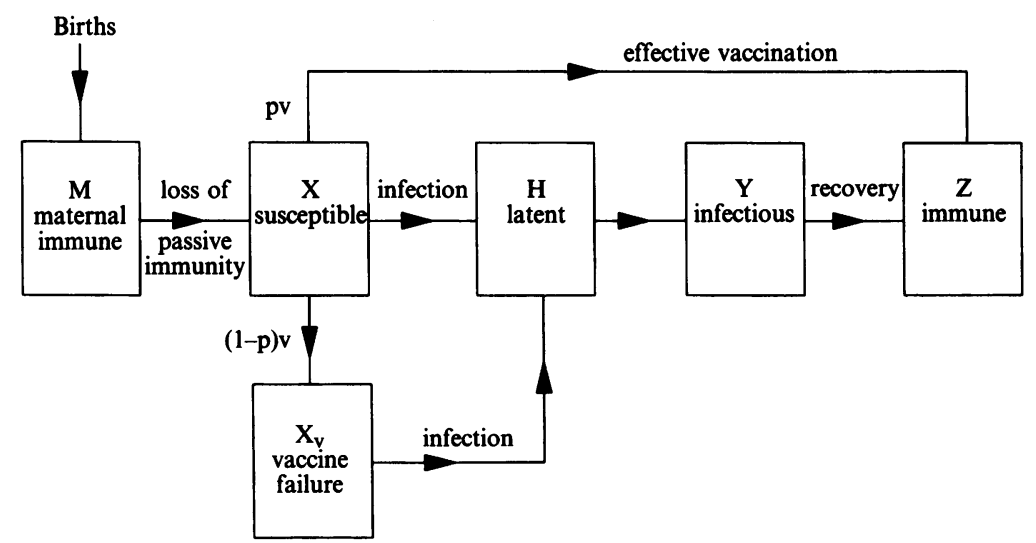

Fig. 1. Measles model. Flow diagram of transitions between population compartments.

infection (class $\mathrm{X}$ ). Susceptibles are removed from class $\mathrm{X}$ as a result of either infection or vaccination. If the former, they move into the latent or pre-patent class, $\mathrm{H}$, prior to becoming infectious to others (class $\mathrm{Y}$ ), finally to recover from infection and establish lasting immunity to reinfection (class $Z$ ). Upon vaccination, a proportion $p$ of susceptibles derive long lasting immunity and move into the immune, $Z$, class. The remaining proportion vaccinated $(1-p)$ are deemed vaccine failures (class $\mathrm{X}_{\mathrm{v}}$ ) who are not protected, and, thus, if exposed to infection can move into the latent class, following the same course as individuals in class $\mathbf{X}$ exposed to infection. Vaccine failures (class $\mathrm{X}_{\mathrm{v}}$ ) may comprise individuals who failed to seroconvert following vaccination and, who are, therefore, seronegative, and individuals serologically positive for measles, yet unprotected if exposed to wild virus. In the current work we assume all individuals develop antibody following vaccination, i.e. all $\mathrm{X}_{\mathrm{V}}$ individuals are seropositive.

\section{Cohort age-structure of model}

Children and adolescents are classified into a number, $n$, of cohorts, where each cohort consists of children born during the same school year (starting 9 September). Individuals aged $n$ and above are classified into a separate adult age compartment, thus the total number of age groupings is $(n+1)$.

Maternal antibody protected newborns enter cohort 1 from births occurring throughout the year. Transition between cohorts only occurs at the end of the year, at which time all the individuals in any one cohort move into the next higher cohort. Thus, mathematically, the model is concerned with rates of change with respect to time in each cohort numbered 1 to $(n+1)$, requiring, simply, a set of ordinary differential equations for each of the six infection compartments shown in Fig. 1, for each of the $n+1$ age groups. In all our simulations, individuals below age 15 are classified into 15 yearly cohorts. Older individuals form the residual compartment numbered 16 where they remain until age 75. A background death rate is assumed for this last age-group only and thus is taken to be $1 / 60$ per person year [9]. The birth rate is fixed at $\mathbf{6 6 6 6 6 6}$ per year which approximates to the annual birth cohort in England and Wales over the time period studied. 


\section{Table 1. Epidemiological parameters used in the model}

\section{Rate parameter}

Force of infection

(/person/year)

\begin{tabular}{|c|c|}
\hline & \\
\hline & $\begin{array}{l}r \text { age } \\
\text { rs) }\end{array}$ \\
\hline & 0.0690 \\
\hline & $0 \cdot 10$ \\
\hline & $0 \cdot 1572$ \\
\hline & $0 \cdot 2005$ \\
\hline & 0.2582 \\
\hline 10 & 0.5167 \\
\hline 15 & $0 \cdot 2549$ \\
\hline & 0.098 \\
\hline
\end{tabular}

Rate of progression from latency (/person/year)

(average duration of latent period, days)

Rate of recovery from infection (/person/year)

(average duration of infection, days)

Rate of loss of passive immunity (/person/year) (average duration of maternal protection, months)
Data/method source

Case notification data/catalytic infection model $[14,19]$

e.g. Household infection/chain binomial method $[20,33,34]$

7

$52 \cdot 14$

e.g. Household infection/chain binomial method $[20,33,34]$

7

$4 \cdot 0$

3

Serological data/exponential decay model $[10,35]$

\section{Age-specific and seasonal contact rates}

Infection of susceptible individuals (classes $X$ and $X_{V}$ in Fig. 1) in any one age class (a single cohort or grouping of cohorts) is dependent upon the effective contact rates with infectious individuals from within their own age class and individuals of each of the other age classes. The term effective contact implicitly assumes the rate of mixing times the transmission probability following contact between infectious and susceptible individuals. These mixing rates are determined from age-specific rates of infection derived from pre-vaccination measles notification data (Table 1) (see Anderson and May for method [19]). The age stratification of these data imposes the age class structure used to define effective mixing patterns in the model, namely, yearly classes up to age 4 years, 5-9 years, 10-14 years and 15 years and above.

The exact form of effective mixing pattern in the population determines a Who Acquires Infection From Whom matrix (i.e. WAIFW matrix [10]), the structure of which is, to a degree, arbitrary because there is no direct quantifiable way of measuring exactly from whom individuals acquire their infection. We have adopted two alternative WAIFW matrices which are epidemiologically plausible and which broadly span the range of possibilities. This will provide an indication of the range of outcomes possible in the results due to different patterns of social interaction. Further discussion of this subject, including the procedure for estimating values of a WAIFW matrix, may be found in references [10] and [20].

The first of these effective mixing matrices, WAIFW 1, has the form shown in the first WAIFW matrix. The 8 by 8 matrix describes the pattern of effective mixing rates (the $\beta$ values) of susceptibles and infectives within and between 8 age classes. The matrix is restricted in the number of different $\beta$ values equal to the 


\begin{tabular}{|c|c|c|c|c|c|c|c|c|c|}
\hline & \multicolumn{8}{|c|}{ First WAIFW matrix } \\
\hline & & $0-$ & $1-$ & $2-$ & $3-$ & $4-$ & $5-$ & $10-$ & $15+$ \\
\hline \multirow{8}{*}{$\begin{array}{l}\text { Susceptible } \\
\text { age class } \\
\text { (years) }\end{array}$} & $0-$ & $\beta 1$ & $\beta 1$ & $\beta 1$ & $\beta 1$ & $\beta 1$ & $\beta 1$ & $\beta 1$ & $\beta 8$ \\
\hline & $1-$ & $\beta 1$ & $\beta 2$ & $\beta 2$ & $\beta 2$ & $\beta 2$ & $\beta 2$ & $\beta 2$ & $\beta 8$ \\
\hline & $2-$ & $\beta 1$ & $\beta 2$ & $\beta 3$ & $\beta 3$ & $\beta 3$ & $\beta 3$ & $\beta 3$ & $\beta 8$ \\
\hline & $3-$ & $\beta 1$ & $\beta 2$ & $\beta 3$ & $\beta 4$ & $\beta 4$ & $\beta 4$ & $\beta 4$ & $\beta 8$ \\
\hline & $4-$ & $\beta 1$ & $\beta 2$ & $\beta 3$ & $\beta 4$ & $\beta 5$ & $\beta 5$ & $\beta 5$ & $\beta 8$ \\
\hline & $5-$ & $\beta 1$ & $\beta 2$ & $\beta 3$ & $\beta 4$ & $\beta 5$ & $\beta 6$ & $\beta 7$ & $\beta 8$ \\
\hline & $10-$ & $\beta 1$ & $\beta 2$ & $\beta 3$ & $\beta 4$ & $\beta 5$ & $\beta 7$ & $\beta 7$ & $\beta 8$ \\
\hline & $15+$ & $\beta 8$ & $\beta 8$ & $\beta 8$ & $\beta 8$ & $\beta 8$ & $\beta 8$ & $\beta 8$ & $\beta 8$ \\
\hline
\end{tabular}

WAIFW 1

Second WAIFW matrix

Infectious age class (years)

$\begin{array}{lccccccccc} & & \text { 0- } & 1- & 2- & 3- & 4- & 5- & 10- & 15+ \\ & 0- & \beta 1 & \beta 1 & \beta 1 & \beta 1 & \beta 1 & \beta 1 & \beta 1 & \beta 8 \\ & 1- & \beta 1 & \boldsymbol{\beta 2} & \beta 1 & \beta 1 & \beta 1 & \beta 1 & \beta 1 & \beta 8 \\ \text { Susceptible } & 2- & \beta 1 & \beta 1 & \boldsymbol{\beta 3} & \beta 1 & \beta 1 & \beta 1 & \beta 1 & \beta 8 \\ \text { age class } & 3- & \beta 1 & \beta 1 & \beta 1 & \boldsymbol{\beta} 4 & \beta 1 & \beta 1 & \beta 1 & \beta 8 \\ \text { (years) } & \beta 1 & \beta 1 & \beta 1 & \beta 1 & \beta 1 & \boldsymbol{\beta} 5 & \beta 1 & \beta 1 & \beta 8 \\ & 5- & \beta 1 & \beta 1 & \beta 1 & \beta 1 & \beta 1 & \boldsymbol{\beta 6} & \beta 7 & \beta 8 \\ & 10- & \beta 1 & \beta 1 & \beta 1 & \beta 1 & \beta 1 & \boldsymbol{\beta} 7 & \boldsymbol{\beta} 7 & \beta 8 \\ & 15+ & \beta 8 & \beta 8 & \beta 8 & \beta 8 & \beta 8 & \beta 8 & \beta 8 & \beta 8\end{array}$

WAIFW 2

number of age classes, i.e. 8 . These 8 mixing rates are distributed in a form which presupposes considerable mixing between age groups as well as within. Thus, for example, it assumes that there is the same degree of mixing within 1-year-olds as between 1-year-olds and most other older age classes (defined as rate $\beta 2$ ). Age groups 5-9 and 10-14 years, the predominantly in-school groups, are marked out as having different (higher in reality) rates of mixing among themselves than with either younger or older age groups ( $\beta 6$ and $\beta 7$ ).

The construction of the second WAIFW matrix, at the other extreme, differs from that of WAIFW 1 in presupposing that the highest rates of contact are predominantly within age groups, with little between age group mixing, i.e. concentrated along the highlighted diagonal.

Values of the contact rates in WAIFW 1 and 2 estimated from pre-vaccination (equilibrium) notification data are, in general, held constant in the model simulations. This implicitly assumes there has been no change in the age-specific mixing pattern in England and Wales since vaccination was introduced in 1968 . However, data from the Department of Education reveal a trend for increase in the percentage of children under 5 attending nursery education, particularly from 1970 onwards [21, 22]. We assume that this secular change in nursery education has increased the rate of mixing within (or within and between) age classes 2-4 years, and investigate its potential influence on measles epidemiology in the vaccination era. In this exercise the relevant values of the mixing matrix (WAIFW 1 and 2) are incremented over the period 1970-92, in proportion to the changes observed in pre-school nursery attendance. Increments are made on a daily, yearly or once-off basis, to mixing rates within, or within and between, age groups 3 and 4 years. 
Mixing between children of primary school age is assumed to be the main contributing factor towards precipitating epidemic (seasonal and longer-term) growth of new measles cases, largely as a result of the high input of susceptible children, previously exposed to relatively low mixing rates, into the school system. As a consequence, seasonal forcing is simulated by switching the mixing rate in age class 5-9 years ( $\beta 6$ in the above matrices), depending on whether school is open or closed for the vacation periods, from its estimated value to one of the (lower) contact rates of the younger age-classes which yields the best fit to the data (set to $\beta 2$ in WAIFW 1 and 2). Simulations are calculated on a daily basis, the school year taken to start on day 252 of the calendar year (9 September), with non-school days, i.e. national holidays and weekends, following the same pattern used by Schenzle [9].

\section{Epidemiological parameter assignments}

Age-specific rates or forces of measles infection prior to the introduction of vaccination have been estimated from age-stratified case notifications for England and Wales pooled for the years 1956-65 [5] using the method described by Anderson and May in 1983 [19]. There is no reliable pre-vaccination ageseroprevalence data from which to measure infection rates. Throughout the period 1956-65 measles incidence was at a dynamic equilibrium, with a very regular biennial epidemicity superimposed on a seasonal periodicity. The averaging of incidence over a number of epidemic cycles provides information which is suitable for deriving information on age-specific transmission rates [14]. The calculated values, recorded in Table 1 , indicate, for each age class, the rate at which susceptibles, $X$ and $X_{V}$, move into the latent class, $H$ (Fig. 1). The per susceptible rate of infection rises to a maximum in the 5-9 year primary school age groups and then declines in adulthood. In the pre-vaccination era the vast majority of cases of measles $(\approx 95 \%)$ were reported in those under 10 years of age. For this reason it should be noted that the accuracy of estimates of the force of infection in the 10-14 year, and 15 and over, age groups is likely to be lower than for the younger age classes (Gay et al., unpublished document). This is of importance when predicting the impact of mass vaccination which has the tendency to increase the average age at infection (both as predicted by mathematical models and as observed).

Other epidemiological rate parameters, such as the average durations of the latent and infectious periods and the rate of loss of maternal antibody protection, are recorded in Table 1, together with data sources and methods of estimation.

\section{Vaccine seroconversion rates, efficacy, and age-specific schedule}

Age-specific rates of vaccination in England and Wales over the period 1968-92 are estimated from published data for England only from the Department of Health records [23] and corresponding birth statistics published by the OPCS [24], based on the method described by Anderson and Grenfell [18]. Suitable vaccination data for England and Wales were not available for the full period required. The schedule was calculated in the following way. The birth cohort each year provides the starting denominator for individuals available for vaccination. 
As each cohort increases in age by one year, the number available for vaccination is reduced by the previous year's number vaccinated. There is assumed to be no effect of mortality. Thus the proportion of unvaccinated individuals in a specified age group who received vaccine in any one year is calculated from the reported number of doses of vaccine administered to that age group in the specified year, divided by the number of individuals in the relevant birth cohort who have not received vaccine in earlier years. For example, if the birth cohort is 666666 , and the recorded number of doses given to this cohort in their first, second and third years of life are 500, 304000 and 158000 , respectively, then the proportion of those previously unvaccinated who receive vaccine in each of these years is $500 / 666666=0 \cdot 001,304000 /(666666-500)=0 \cdot 456$, and $158000 /(666666-500$ $-304000)=0 \cdot 436$. Where cohorts are grouped together in the official figures, an equal distribution across the cohorts has been assumed. With the introduction of MMR vaccine in October 1988 individuals in the 4-15 year age groups were offered MMR triple vaccine irrespective of previous vaccination history for measles. Thus, in calculating the proportion of each age group receiving the vaccine in the years after MMR was introduced, the full birth cohort for each cohort between 4 and 15 years of age was assumed to be available for vaccination in the first year and progressively reduced each successive year by the numbers receiving MMR. Official statistics show the distribution of MMR vaccine in 1989/90 for the 4-7 year age-group [23]. Making the assumption that this distribution has not altered since then, it is used as a template for the administration of vaccine by age from 1989 onwards.

The calculated rates are imposed in the model on the susceptible class, $X$ (Fig. 1), as instantaneous rates over calendar years and on a cohort basis [18], to recover the impact of mass vaccination since it began in England and Wales. It should be noted that changes in the method of recording vaccination uptake, and the change over from single antigen measles to MMR vaccine [23], are likely to have affected the accuracy of reports on vaccine coverage rates.

We define vaccine efficacy as the proportion of a previously uninfected group of individuals (class $X$, Fig. 1) who, when vaccinated, are no longer able to transmit the infection (class $Z$, Fig. 1). The value of this vaccine efficacy, $p$, is set at $90 \%$. This is at the lower limit of most published estimates, which are based upon clinical diagnosis [25] but appears realistic from recent studies conducted by the Communicable Disease Surveillance Centre, based upon laboratory confirmed reported cases $[15,26,27]$. The proportion of susceptibles who, following vaccination, remain able to transmit the infection, $1-p$, enter the vaccine failure class, $\mathrm{X}_{\mathrm{V}}$ (Fig. 1). We make the simplifying assumption that the measles vaccine seroconversion rate is $100 \%$, i.e. all susceptibles given vaccine produce detectable, lifelong, specific antibody. As a consequence, all those in the $X_{v}$ class are seropositive. This yields an upper limit on model estimates of seroprevalence, but does not alter the transmission dynamics.

\section{Seroprevalence data}

In verifying the predicted impact of vaccination on measles epidemiology we make use of age-stratified seroprevalence surveys from England and Wales over the period 1969-91. Data for 1969 derive from serum samples collected at the 
Northern General Hospital, Sheffield (see Walker et al. [28] for further details on sample composition and collection). Sera were screened by indirect ELISA using the method described by Cox [29]. During the period 1979-83 serum samples were collected from 9 Public Health Laboratories in England, and screened by the Central Public Health Laboratory and National Institute of Biological Standards and Control using the measles haemagglutination inhibition (HI) test (data unpublished in full [10]). The PHLS countrywide surveillance study of MMR antibodies, in which samples have been collected from a number of PHLS microbiology laboratories from England and Wales, provides a source for measles age-prevalence data for 1986 and 1991. Samples were screened by HI and equivocal results confirmed by indirect ELISA [16]. In each survey the sera were a representative selection of those submitted to the laboratories for routine virological investigation. Standardization of cut-off points for seropositivity, other than for 1986 and 1991, was not possible. Sample sizes for unpublished surveys are given in the relevant figure legends.

\section{RESULTS}

\section{Capturing the dynamics of pre-vaccination measles}

Weekly case notifications reported to OPCS over the period 1956-65 are used to calculate the average biennium ( \pm 1 standard deviation) shown in Fig. 2 (squares and bars). The raw data were first scaled to account for under-reporting based upon the findings of Fine and Clarkson, 1982 [8], i.e. $32 \%$ under-reporting in major epidemic years, and $42 \%$ under-reporting in minor epidemic years, with an average of $36 \%$. These observations are compared with the model simulations using either WAIFW 1 or WAIFW 2, as detailed in Fig. 2.

The scaled notifications clearly show alternate years of a minor epidemic followed by a major epidemic. Each is punctuated by three main dips in incidence which correspond in time to the three major holiday periods of the school calendar. The start of each major epidemic can be timed to just after the start of the new school year around week 39 of each biennium (September of each year). It is clear that the model mirrors these events well with either format of effective mixing. The model over-estimates incidence of infection in the first 2 terms of each minor epidemic (September to Easter) when reporting is at its lowest. It is possible that during this period reporting efficiency is lower than previously suggested by Fine and Clarkson [8].

A comparison between the average age-distribution of cases reported (scaled) over the period 1956-65 and the model output for each form of mixing pattern is recorded in Fig. 3. The figure indicates a good agreement between observation and prediction throughout the age range.

\section{The impact of mass vaccination: comparison between prediction and observation}

The ability of the model to simulate the impact of measles mass vaccination is evaluated in Figs 4-6. The time and age-dependent vaccination schedule is applied to the model and the results compared with 3 types of observational data over the period since the start of vaccination in 1968 to the present day. 


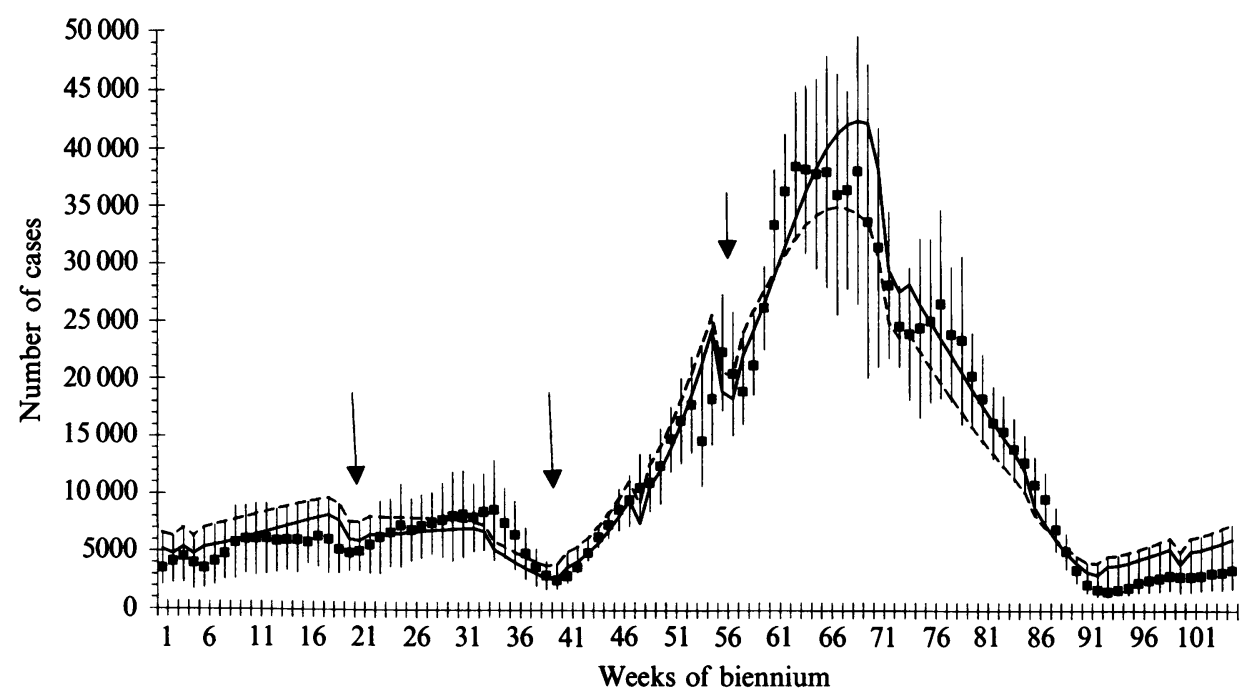

Fig. 2. Comparison of weekly measles notifications in England and Wales in an average biennium for the period 1956-65 (solid square markers \pm 1 standard deviation) with model output using 2 different effective mixing matrices. WAIFW 1 shown as solid line, WAIFW 2 as dashed line. Arrows indicate approximate timing of start of school terms in 52 week period. Week 1 begins 1 January.

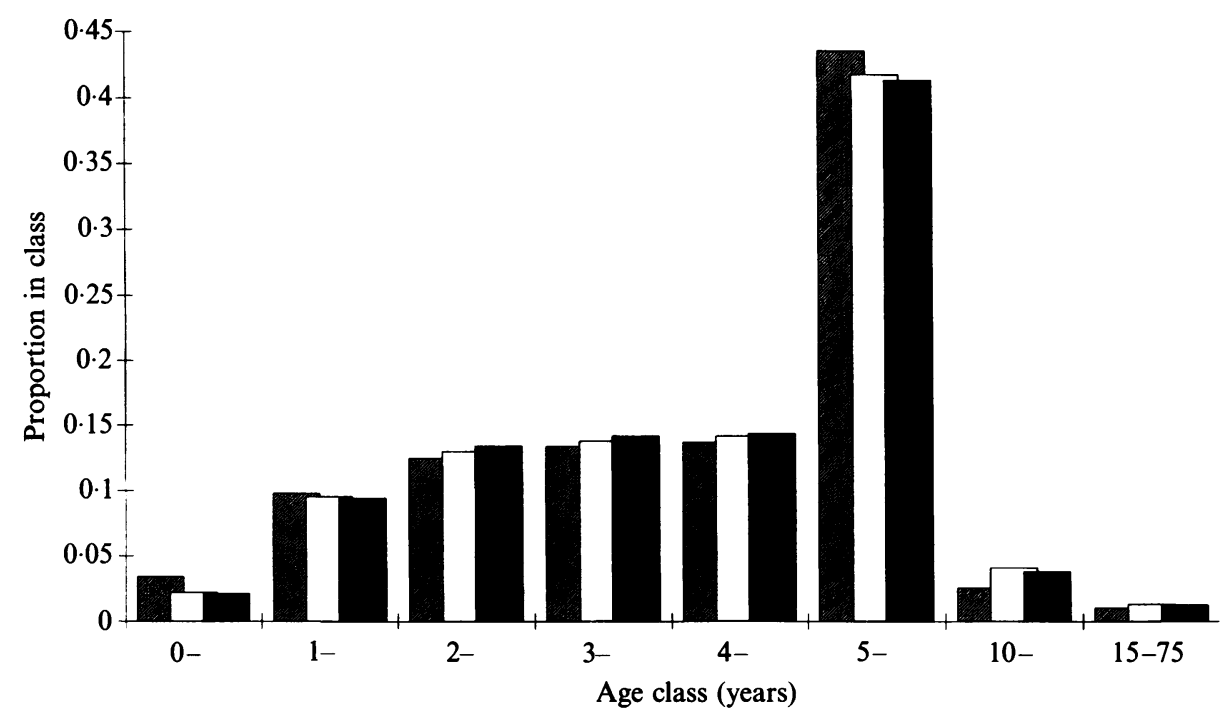

Fig. 3. Comparison of the observed age distribution of measles cases in England and Wales averaged over the period 1956-65 (hatched bars), with model prediction, using WAIFW 1 (open bars), or WAIFW 2 (solid bars).

In Fig. 4 the quarterly number of measles cases predicted using WAIFW 1 (dashed line) and WAIFW 2 (dotted) from 1963 to 1993 are compared with the quarterly reports for measles from OPCS. The reports are scaled by a factor $\mathbf{1 . 5 6}$ to account for an assumed under-reporting level of $36 \%$. Once vaccination 


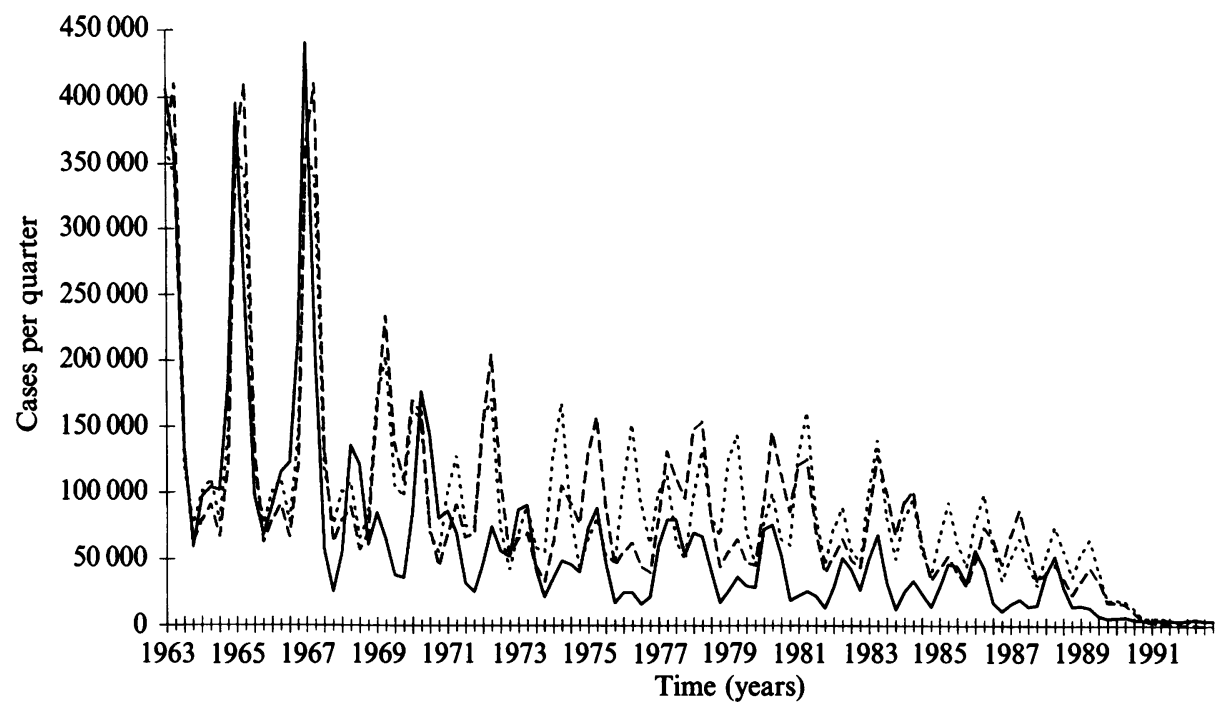

Fig. 4. Temporal incidence of measles from 1963 to 1993 . Observed quarterly notifications in England and Wales (solid line) compared with model output using WAIFW 1 (dashed line), and WAIFW 2 (dotted line). National vaccination introduced in 1968.

commences in 1968 the predicted cases fall out of phase from the observed. Furthermore, predicted incidence is significantly higher than that observed through to 1990. After 1988, with high MMR vaccination, prediction and observation show a similar trend towards negligible levels in the early 1990 's.

Fig. 5a-d illustrates the comparison of age-prevalence profiles for measles specific antibodies with the predicted seroprevalence profiles from the model using WAIFW 1 (solid line) and WAIFW 2 (dashed line) at four time points, 1969, 1979-83 (year 1981 of model), 1986 and 1991. The model output records the total proportion of individuals, by age, in classes $M$ (those with maternal antibody), $Z$ (immunes) and $\mathrm{X}_{\mathrm{V}}$ (seroconverted vaccine failures), all of whom would be recorded as seropositive in a survey. This represents a slight over-estimate of the true level of seroprevalence by ignoring those who do not seroconvert following vaccination.

In general, there is a high degree of concordance between model prediction and observed seroprevalence (Fig. 5). There is a tendency for the model with greater within group mixing (WAIFW 2) to provide a better fit at higher seroprevalence in older children and teenagers. Fig. $5 a, b$ for 1969 and 1979-83 reveals a slight tendency for model predictions to underestimate observed seroprevalence in the age range 3-8 years. In 1986, after approximately 18 years of mass cohort immunization, the model (with either form of mixing) yields a significantly lower estimate of prevalence in the 3-10 year age range than was observed (Fig. $5 c$ ). The deficit seen in 1986, is, however, largely recovered by 1991 (Fig. $5 d$ ), roughly 3 years into the mass MMR vaccination campaign. Over the period 1988-91 a significant increase in level of immunization of children by 2 years of age had been achieved $[23,30]$ and a catch-up programme of vaccination of, particularly, 4- and 5 -year-olds had been undertaken [1].

There is evidence of a trough of susceptibles of primary school age children 

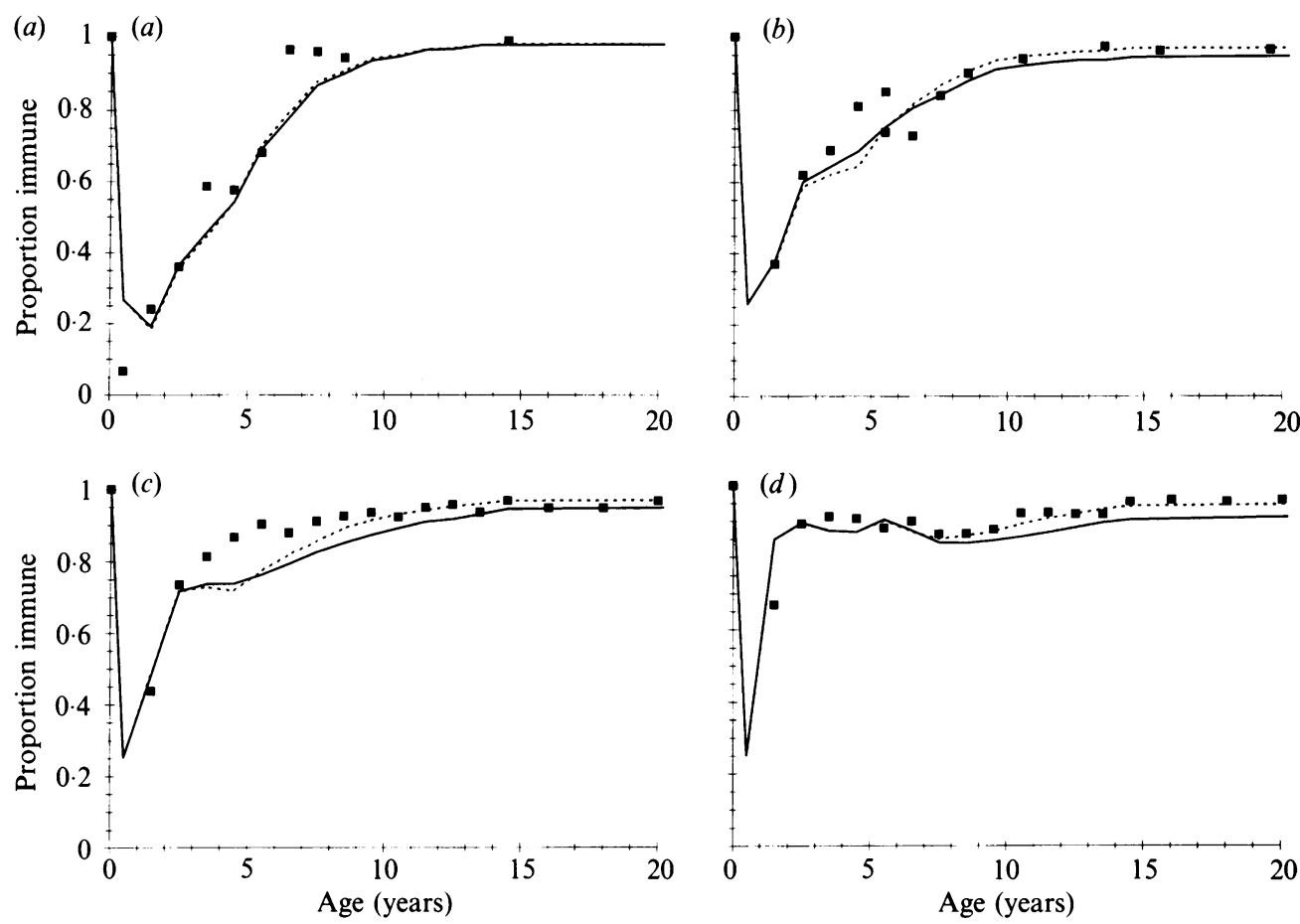

Fig. 5. Age-seroprevalence profiles. Data from surveys in 1969 (graph $a$ ), 1979-83 (graph $b$ ), 1986 (graph $c$ ) and 1991 (graph $d$ ) shown as solid square markers are compared with model output for the years 1969, 1981, 1986 and 1991, respectively, using WAIFW 1 (solid line) and WAIFW 2 (dashed line). Age classes (mid-point in years) and sample sizes for unpublished survey data are as follows: $1969: 0.5,30 ; 1.5$, $54 ; 2.5,64 ; 3.5,29 ; 4 \cdot 5,33 ; 5 \cdot 5,22 ; 6 \cdot 5,29 ; 7 \cdot 5,25 ; 8 \cdot 5,18 ; 14 \cdot 5,108.1979-83: 1 \cdot 5,35$; $2 \cdot 5,142 ; 3 \cdot 5,120 ; 4 \cdot 5,118 ; 5 \cdot 5,95 ; 5 \cdot 5,453 ; 6 \cdot 5,111 ; 7 \cdot 5,61 ; 8 \cdot 5,96 ; 10 \cdot 5,1512 ; 13.5$, $364 ; 15 \cdot 5,227 ; 19 \cdot 5,851 ; 23 \cdot 5,200.1991: 1 \cdot 5,208 ; 2 \cdot 5,223 ; 3 \cdot 5,206 ; 4 \cdot 5,195 ; 5 \cdot 5,201$; $6 \cdot 5,210 ; 7 \cdot 5,192 ; 8 \cdot 5,170 ; 9 \cdot 5,213 ; 10 \cdot 5,169 ; 11 \cdot 5,188 ; 12 \cdot 5,214 ; 13 \cdot 5,189 ; 14 \cdot 5,229$; 16,$263 ; 18,557 ; 20,504$.

(roughly 6-11 years of age) in 1991 (Fig. 5d), which represents the group of individuals too old to have been covered by the MMR immunization programme, and who are now experiencing a very low rate of exposure to wild virus (as predicted would be the case [20]).

Fig. 6 provides a comparison between the proportion of cases in each age class reported to OPCS, for years complementary to the serological data, and the proportions predicted by the model. The agreement is, in general, good, although the fit is markedly better using the model with greater between group mixing (WAIFW 1). In general there is a tendency to under-estimate the proportion of cases in the youngest age groups $(0-4$ years), and, therefore, over-estimate proportions in the other age groups. By 1991 (Fig. 6d), at which time overall incidence has declined to very low levels following the introduction of MMR vaccine (Fig. 4), the observed proportions of cases in the first and second years of life are markedly higher than those predicted.

In a recent investigation, Brown and colleagues [15] identified a significant degree of inaccuracy in the clinical diagnosis of measles cases reported in the UK 

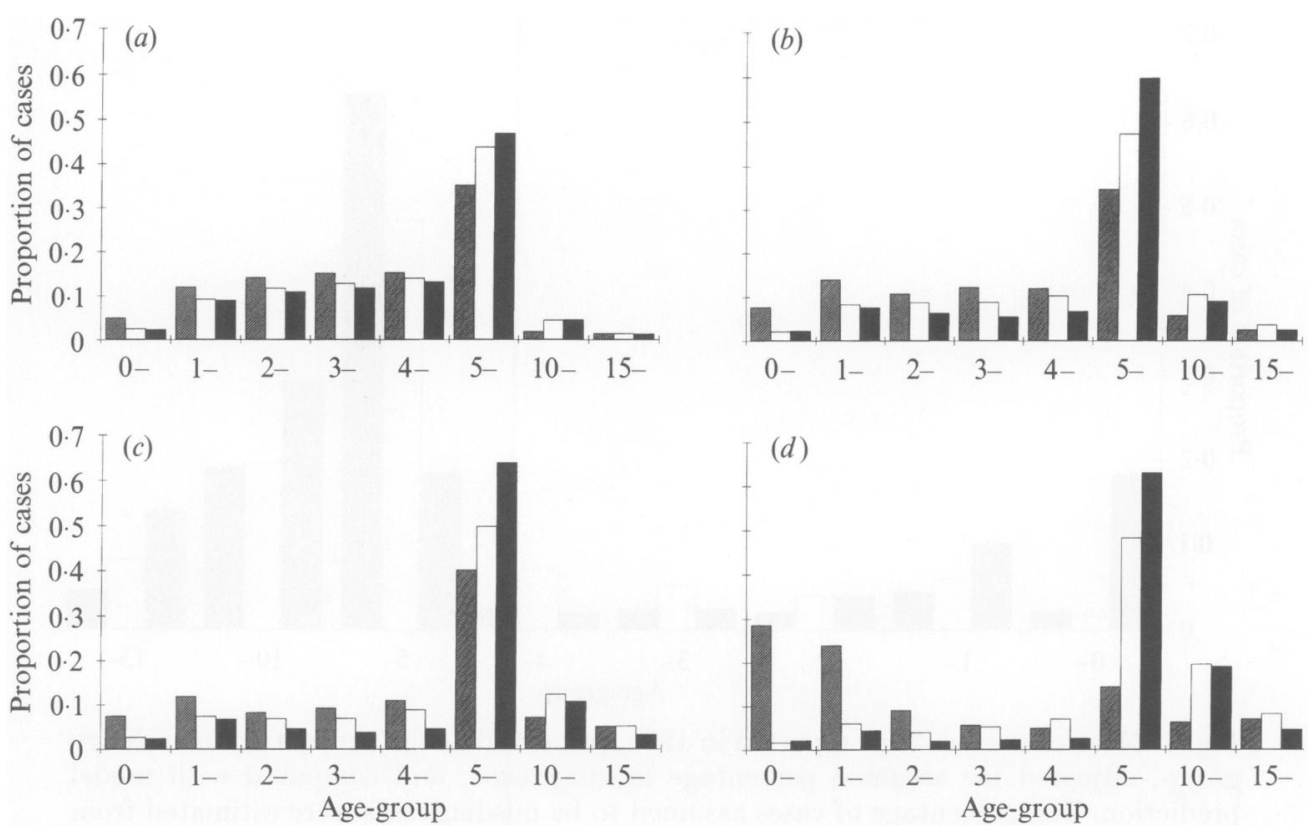

Fig. 6. Age-stratified measles notification data (as a proportion of the total in each age class) reported in England and Wales in the years 1969, 1981, 1986 and $1991(a-d$, respectively), compared with model output, using two different mixing matrices, for the corresponding years. Other details as in Fig. 3.

between 1991 and 1993. The proportion of cases which could be confirmed by IgM detection in serum was dependent upon age, with particularly low accuracy in those under 4 years. Fig. 7 shows the same data as for Fig. $6 d$, except that the 1991 notified cases have been scaled by the proportion assumed to be misdiagnoses according to the above study. The fit of the model is improved. However, the model is still over-estimating cases in the 5-9 year age group and under-estimating cases in those aged less than 1 year and 15 years and over.

\section{The impact of secular change of pre-school education}

Over the period 1970-92, the proportion of 3- and 4-year-olds in state-registered nurseries has risen from roughly 10 to $50 \%$ [21]. This observation raises the possibility that increments in the rate of mixing within age groups 3 and 4 (cohorts 4 and 5 in the model - see WAIFW 1 and 2) over the last two decades may be a contributing factor in the under-estimation by the model of observed seroprevalence in children aged 2-10 years in 1986 (Fig. 5c) and of the observed proportion of cases in pre-school age groups (Fig. 6).

Implementing changes to the relevant contact parameters in WAIFW 1 and 2 over the period 1970-92 was unable to improve significantly the model fit to the 1986 seroprevalence data, without dramatically perturbing the incidence of infection. However, in all instances a better agreement between predicted and observed proportions of cases in age groups $2-4$ years was achieved. The importance of secular change in pre-school mixing patterns in measles epidemiology remains unclear. 


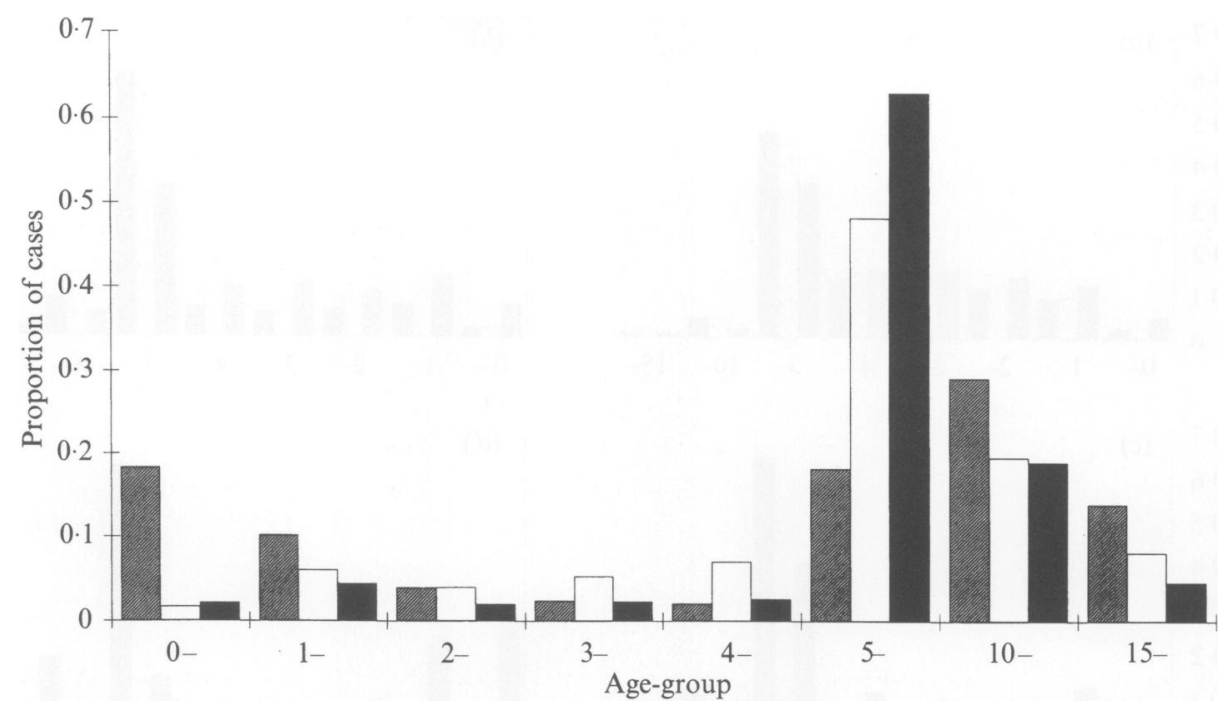

Fig. 7. Measles notifications reported in 1991, presented as the proportion in each age group, adjusted for assumed percentage misdiagnosed, and compared with model prediction. The percentage of cases assumed to be misdiagnoses were estimated from Brown and colleagues 1994 [15], and, for each age group (years), are: $<1,88.9 ; 1-4$, $92.6 ; 5-9,78 \cdot 0 ; 10-14,20 \cdot 0 ; 15$ and over, $41 \cdot 7$. Other details as for Fig. $6 d$.

\section{Projections of the impact of proposed policies}

At current levels of vaccination and with efficacy set to $90 \%$ the model predicts measles infection to be virtually eliminated from England and Wales in 1992/3 (Fig. 4). This stems largely from the increased levels of uptake brought about by the introduction of MMR in late 1988. Whilst this result does not exactly match with measles notifications in England and Wales, the observed trend is highly consistent with model predictions. Discrepancies between prediction and observation may be accounted for by countrywide variation in (i) vaccination coverage, and (ii) population density and structure, which could allow for continued chains of transmission, but which are not incorporated into the model structure.

In the light of these observations, there are 2 aims of any changes in policy in England and Wales. First, in the short term a catch-up vaccination programme might be implemented to accelerate the impact of the current policy, elevating levels of herd immunity where deficient and cancelling out the effects of variations in compliance across the country and lower coverage in the past. For the longer term, however, it is necessary to decide whether, given time, the current policy of a single dose is sufficient to eliminate infection. If not, then there is a need to explore strategies which aim to offset this deficiency, elevating levels of herd immunity above that which could be achieved by a single dose.

\section{Vaccination strategies investigated}

The following vaccination strategies for measles might be considered for the future in England and Wales, as summarized in Table 2.

From 1994 onwards we establish a base-line policy for England and Wales in which the current level of $92 \%$ measles vaccination by the end of the second year 


\title{
Table 2. Description of vaccination strategies investigated
}

\author{
Strategy option \\ Description \\ Baseline \\ $92 \%$ coverage of boys and girls during second year of life. \\ Vaccine efficacy $90 \%$. \\ Campaign \\ Baseline policy plus one-off campaign in which $50 \%$ of target group \\ are vaccinated over 2 -month period in 1994 . Policy may be selective \\ or unselective. \\ Target groups : \\ (i) pre-school 1-4 years old \\ (ii) primary school 5-11 years old \\ (iii) all school age children, i.e. 5-16-year-olds \\ Two-dose Baseline policy plus a second dose in which $50 \%$ of target group \\ receive vaccine. Policy may be selective or unselective. Begins 1994 \\ Target groups : \\ (i) 4-year-olds \\ (ii) 11-year-olds \\ Pulse $\quad$ Baseline policy plus a campaign once every 5 years in which $50 \%$ of \\ individuals in the target age range (5-11-year-olds) receive \\ vaccine. Begins 1994.
}

of life [23] is maintained. All subsequent policies are assumed to be in addition to this basic level of vaccination. In general, simulations assume a vaccine efficacy of $90 \%$, although we explore the sensitivity of results for a higher vaccine efficacy of $95 \%$.

As a means of accelerating the impact of high levels of coverage now being achieved through routine MMR immunization, and to offset the risk of an epidemic occurring in age groups with immunity deficits identified in seroprevalence data (i.e. 6-11-year-olds, Fig. 5), a one-off vaccination campaign is undertaken during two months in 1994, as a result of which $50 \%$ of susceptibles in the target age groups are actually vaccinated. Various options are explored, namely, (i) targeting pre-school children only (1-4-year-olds), (ii) targeting primary school children only (5-11-year-olds), or (iii) targeting all school age children (5-16-year-olds).

Two types of longer-term measures to increase herd immunity levels above those possible by a single dose routinely administered in the second year of life are explored. The first of these is a 2-dose schedule, in which the second dose is targeted at a specific age group on a continuous basis. In the simulations we consider the alternative target ages of 4 and 11 years, corresponding to the delivery opportunities created by the existing schedule for booster DT (diphtheria, tetanus) doses given to pre-school children and selective schoolgirl rubella vaccination, respectively [31]. Again it is assumed that $50 \%$ of susceptibles are successfully reached by this approach.

Additionally, vaccination is assumed to be either selective, i.e. only children without a record of vaccination are offered vaccine, or unselective in which any child of the target age is eligible. In terms of the model structure, selective implies delivery of vaccine only to susceptibles in class $\mathrm{X}$, whereas, unselective vaccination results in delivery to any susceptible, whether in class $X$ or class $X_{v}$. The importance of this distinction is that an unselective vaccination policy has the potential to reach vaccine failures $\left(\right.$ class $\left.\mathrm{X}_{\mathrm{V}}\right)$. 
The second of 2 longer-term measures investigated is the delivery of vaccine across a wide age range every few years. Specifically, primary school-children (aged 5-11 years) are offered vaccine once every 5 years. The rationale behind this pulse vaccination policy has been the subject of a recent study [32] and in principle rests upon the idea of preventing the build-up of susceptibles in the high contact rate age classes (school ages) so important in driving epidemic cycles, and regularly suppressing susceptible numbers below epidemic proportions. Again a $50 \%$ success rate is assumed, and the policy may be either selective or unselective. It is appropriate to vaccinate every 5 years, being the time it takes for all the preschool group at the time of the campaign to enter schooling.

The success rate of $50 \%$ used in all these simulations is for illustrative purposes only and is not meant to indicate the level expected. The successful vaccination of $50 \%$ of all susceptibles is not necessarily equivalent to a coverage of $50 \%$. For an unselective policy, in which vaccine is offered to any eligible child, it might take $95 \%$ coverage in reality to reduce susceptibility by $50 \%$. The relationship between coverage and vaccine uptake by the target population will be markedly dependent upon the behavioural characteristics of the target group: there may be some who are beyond the reach of the services and can never be vaccinated, or alternatively, mothers of children who have received vaccine in the past may be reluctant to agree to the administration of a second dose ( $\mathrm{N}$. Calvert, personal communication).

\section{Predicted impact of future vaccination policies}

In the following section we utilize the mathematical model to investigate a variety of options for change in the existing measles vaccination policy in England and Wales. In all simulations WAIFW 1 is adopted, since this form of mixing provided the best overall fit to observed data. Secular changes in rates of mixing in pre-school children are not considered further.

Low level incidence (predicted and observed) in England and Wales today severely limits the usefulness of case rates as an outcome variable. Thus, to provide an indication of the relative merits of different vaccination strategies from 1994 onwards we present trajectories of the Effective Reproduction Number, $R$, which is a measure of the potential of the infection to spread in the population. $R$ defines the average number of secondary cases generated for each primary case in a partly immune population, and, simply, may be considered a weighted average reproduction number for all age classes. If $R>1$ then incidence will rise, or, put another way, an epidemic can occur. If $R<1$, then incidence will decrease and an epidemic is unlikely. The details of estimation of the effective reproduction number follows that presented by Anderson and May, 1991 [20], and more recently by Gay and colleagues (unpublished document).

Fig. 8 plots trajectories of $R$ over the period 1985-2015 for the baseline policy based on two assumptions of vaccine efficacy; $90 \%$ (solid lines) and $95 \%$ (dashed lines). Two measures of the effective reproductive rate are recorded. First, a maximum for each year, $R_{\max }$ (thick lines in Fig. 8), estimated at the start of each school year after the summer holidays (9 September), when the potential for a seasonal epidemic is at its highest (note the timing of the major epidemic in each biennium prior to mass vaccination in Fig. 2). Second, an average value of the 


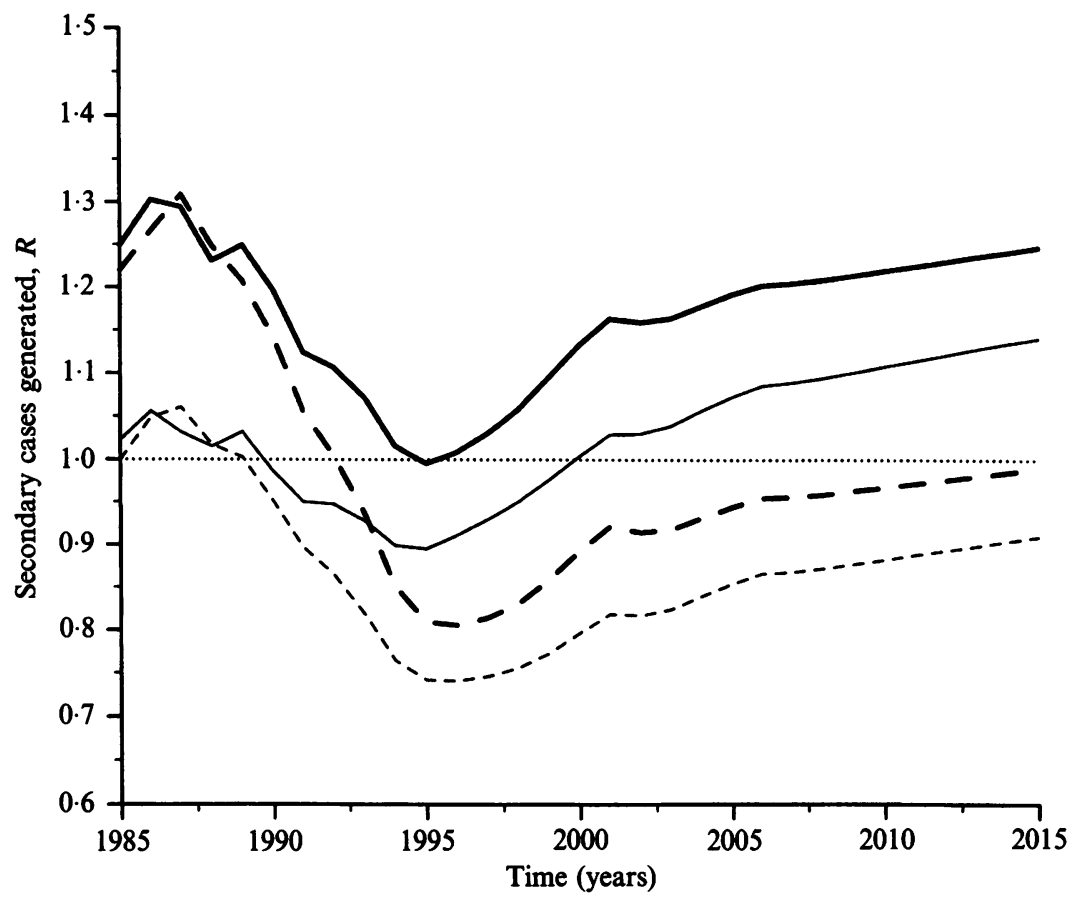

Fig. 8. Baseline policy of $92 \%$ coverage by age 2 years. Trajectories of the effective reproductive number, $R$, under 2 levels of assumed vaccine coverage, $90 \%$ (solid lines) and $95 \%$ (dashed lines). Two forms of $R$ are shown; $R_{\max }$ (thick lines) which records the maximum transmission potential for each year, and $R_{\mathrm{avg}}$ (thin lines), the average reproductive potential of the infection each year.

effective reproductive rate throughout a year, $R_{\text {avg }}$ (thin lines in Fig. 8), which provides a measure of the potential for infection persistence, i.e. $R_{\mathrm{avg}}<1$ leads to elimination, $R_{\text {avg }} \geqslant 1$ indicates persistence. Prior to the introduction of MMR vaccine in late 1988, the value of $R_{\max }$ (Fig. 8 , thick lines) is considerably greater than unity, indicating a high potential for seasonal epidemics. However, the effective reproductive rate will vary throughout the year, as susceptible numbers decline and as mixing rates alter in time with the opening and closing of schools. As would be expected, the value of $R_{\text {avg }}$ prior to 1988 is around unity (Fig. 8, thin lines), indicating stable endemic persistence.

Over the early period of MMR immunization (1988-95) high-level coverage leads to a decrease in the transmission potential and the value of $R_{\text {avg }}$ rapidly falls below unity (Fig. 8, thin lines). This inevitably causes a declining trend in incidence and, eventually, to the elimination of infection as illustrated in Fig. 4. Concomitant with the decline in incidence, herd immunity is increasingly sustained by vaccination alone, with the result that susceptible numbers begin to increase (partly as a consequence of vaccine failures). This is illustrated in Fig. 11 $a$, which shows changes in the susceptible fraction of the population over the period 1985-2015 for $90 \%$ vaccine efficacy. Susceptibles are stratified into unvaccinated (class $\mathrm{X}$ ) and vaccine failures (class $\mathrm{X}_{\mathrm{v}}$ ). The consequence of this increase in susceptibles is a rise in $R_{\text {avg }}$ after 1995 (Fig. 8), which, for $90 \%$ vaccine efficacy, exceeds unity (the threshold for persistence) from the year 2000 (solid thin line), 


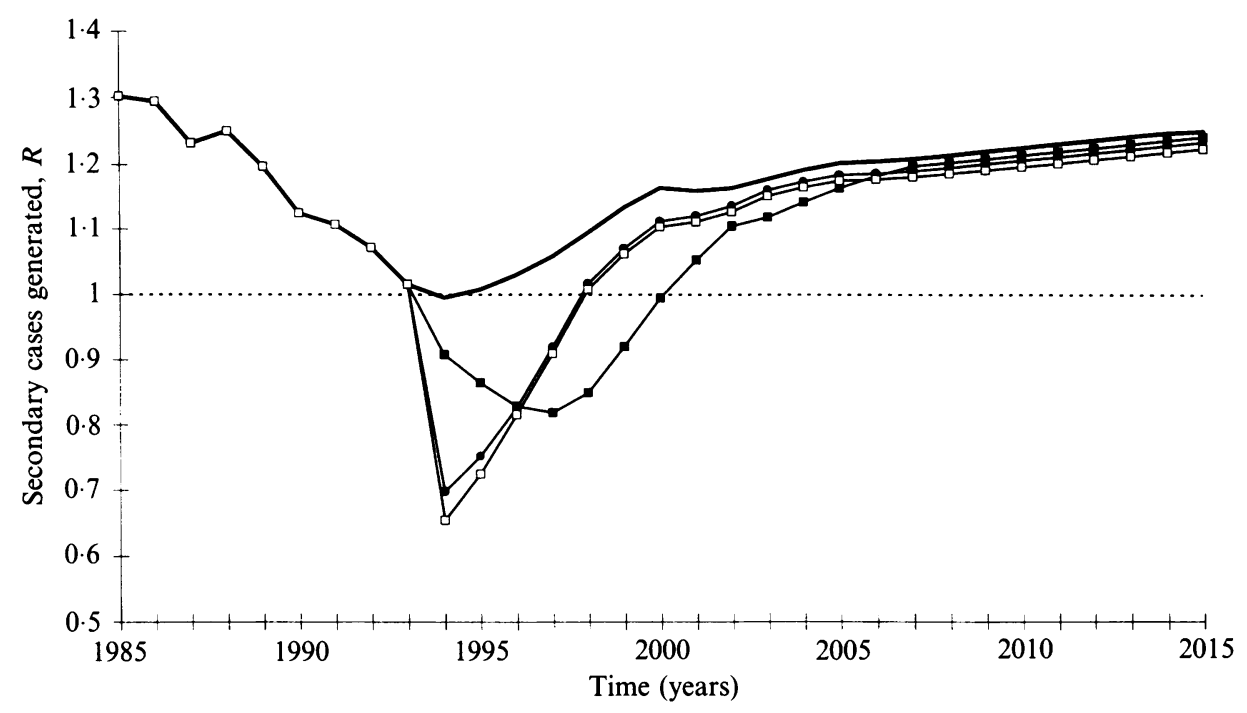

Fig. 9. Relative merits of campaign strategies (unselective), targeted at different age groups, compared with a baseline policy of $92 \%$ coverage by age 2 years. The Effective Reproduction Number, $R$ (the average number of secondary cases generated for each primary case) is plotted over time from 1985 to 2015 . For $R$ greater than the threshold of 1 there is potential for an epidemic to occur. Target age groups: under 5 years (filled squares), primary school ages 5-11 (filled circles), all school age, 5-16 years (open squares). Thick solid line shows baseline policy (vaccine efficacy $90 \%$ ).

or, for $95 \%$ efficacy, remains below unity until after 2015 . The sensitivity of the results to vaccine efficacy illustrates the importance of vaccine failures to the persistence of infection at these high levels of vaccine uptake.

Changes in the value of $R_{\max }$ (Fig. 8, thick lines) following 1988 are shown to track those of $R_{\text {avg }}$ for each of the two assumed vaccine efficacy levels. At $90 \%$ efficacy the potential for seasonal epidemics, defined by $R_{\max }>1$, remains throughout the time period shown in Fig. 8 (thick solid line) barring 1995. At $95 \%$ efficacy $R_{\max }$ falls below unity in the early 1990 s and remains so until after 2015 .

Thus, if we assume a vaccine efficacy of $90 \%$, the model predicts, at the level of coverage currently achieved (around $92 \%$ by age 2 years), the potential for measles persistence $\left(R_{\mathrm{avg}}>1\right)$ will be temporarily interrupted in the $1990 \mathrm{~s}$ but return thereafter, and the threat of seasonal outbreaks $\left(R_{\max }>1\right)$ will continue almost unchecked. What, therefore, are the merits of the different policy options available? In all subsequent simulations a vaccine efficacy of $90 \%$ is adopted, and, for clarity, comparisons are made of the predicted values of $R_{\max }$.

Fig. 9 shows the impact of 3 different campaign policies (dashed lines) in 1994 compared with the baseline policy alone (thick solid line, $90 \%$ efficacy). Where $R$ exceeds the threshold unity line the potential for epidemics exists. Campaigns are targeted at all pre-school (1-4-year-old), primary school (5-11-year-old) or all school age (5-16-year-old) children. Each of the three schedules has the effect of lowering the transmission potential of measles, which, in practice, will limit the likelihood of outbreaks due to countrywide heterogeneities in population density and vaccination coverage. However, targeting vaccine at school-age children will have the most immediate results, since it is here that immunity gaps remain (Fig. 


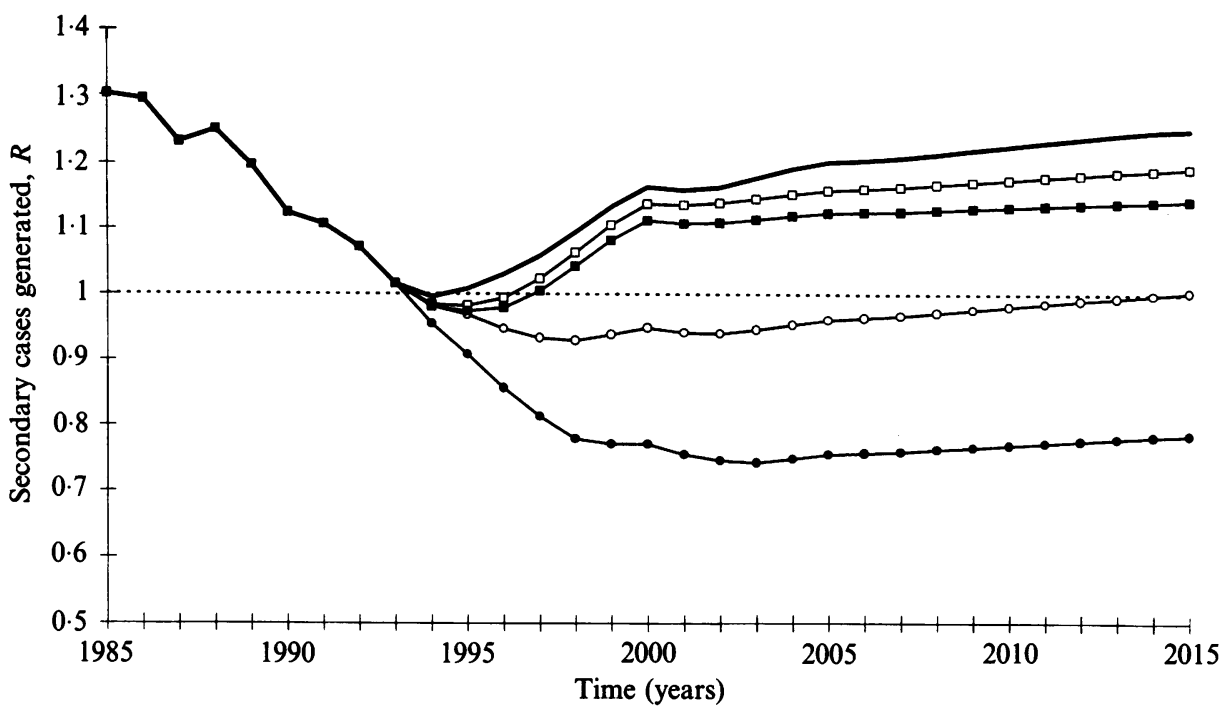

Fig. 10. Relative merits of 2-dose policies targeted at different age groups compared with the baseline policy (thick solid line; $90 \%$ vaccine efficacy). Target age of 11 years (squares), or 4 years (circles). In each case the second dose is administered either selectively to those without a previous vaccination record (open markers), or unselectively (filled markers).

5) and where contact rates are maximum. Including secondary school children in a campaign appears to provide limited benefit above that obtained from primary school immunization alone. Two caveats surround this prediction. First, as each year passes the trough of susceptibles in the 5-11 year group will be moving into the secondary school ages. Second, estimates of the mixing rates within secondary school children are less reliable than for younger ages and their contribution towards future outbreaks is difficult to calculate. For these reasons, it would appear more sensible to consider both primary and secondary school children in a campaign policy.

For each type of campaign the impact is short-lived, since such a policy does not address the problem that current levels of routine immunization fail to prevent the build-up of susceptible children who have escaped vaccination or failed to gain protection from vaccine. There is a delay of a few years in the impact of the preschool campaign, compared with school-age vaccination, as the highly protected young cohorts gradually move into the high contact rate school age classes.

Fig. 10 illustrates the impact of targeting 4-year-olds or 11-year-olds for a second dose. Results are shown for vaccine delivery that is either selective (given to those who have never received vaccine) or unselective (independent of vaccination history). It is clear that a second dose early in life is optimal compared with a later age. This is simply because for such a highly transmissible infection as measles the number of susceptibles allowed to remain between ages 1 and 11 will rise to proportions sufficient to maintain infection. It is clear that vaccinating irrespective of vaccine history offers greater benefit. Fig. 11 expands upon this point and hows the relative proportions of vaccine failures and unvaccinated susceptibles. In the top graph (Fig. 11a) under the baseline policy, the proportion susceptible up to 1988 (MMR introduction) is approximately $6 \%$, of which only 

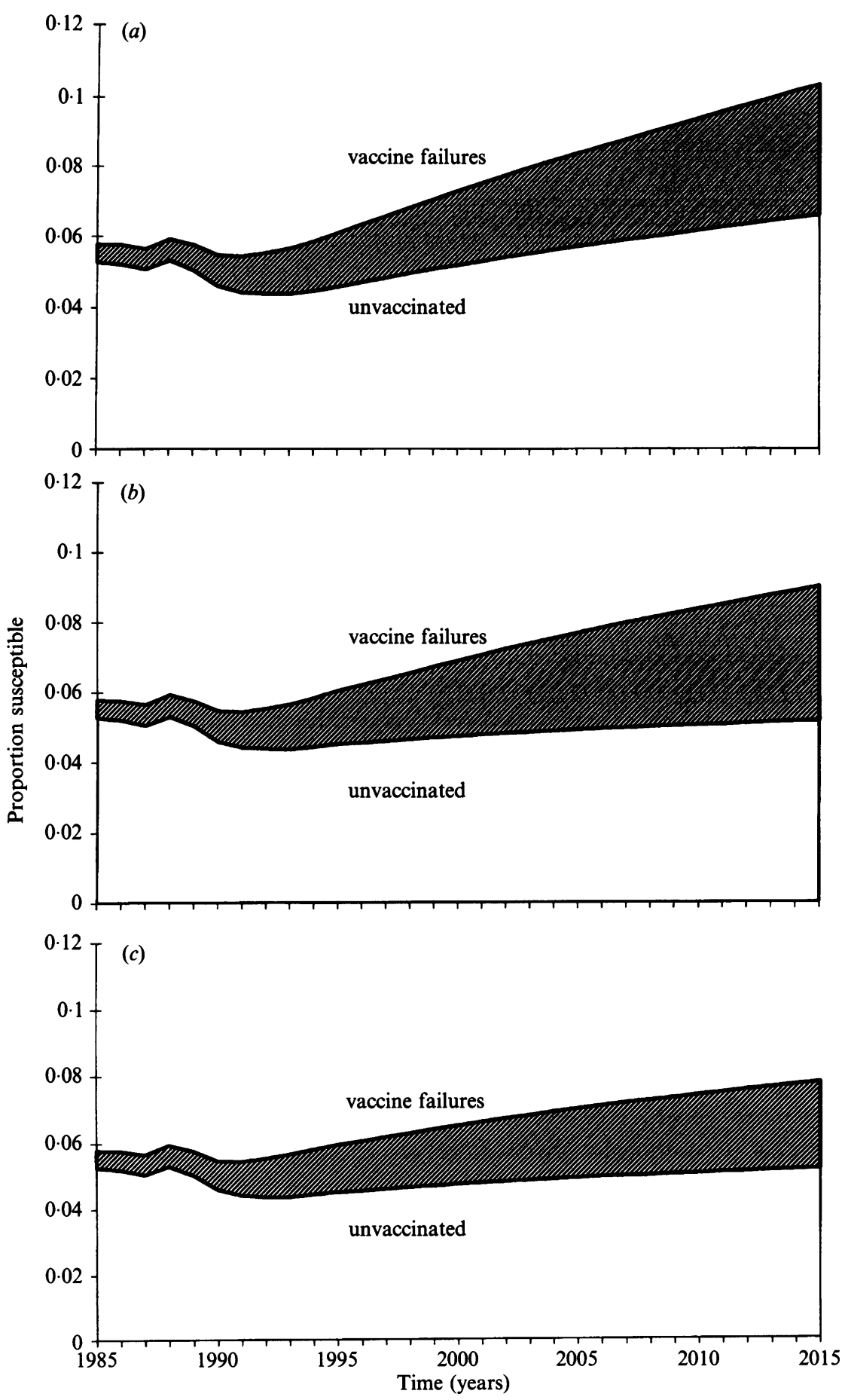

Fig. 11. Measles susceptibility, stratified into proportion unvaccinated and vaccine failures (diagonal shading), over time, for different immunization strategies. Graph $(a)$ shows the results for the baseline policy alone (vaccine efficacy $90 \%$ ), graph $(b)$ for a 2 -dose policy (second dose at age 4 years) selectively given to those without vaccination history, and graph $(c)$ for an unselective 2-dose strategy. 


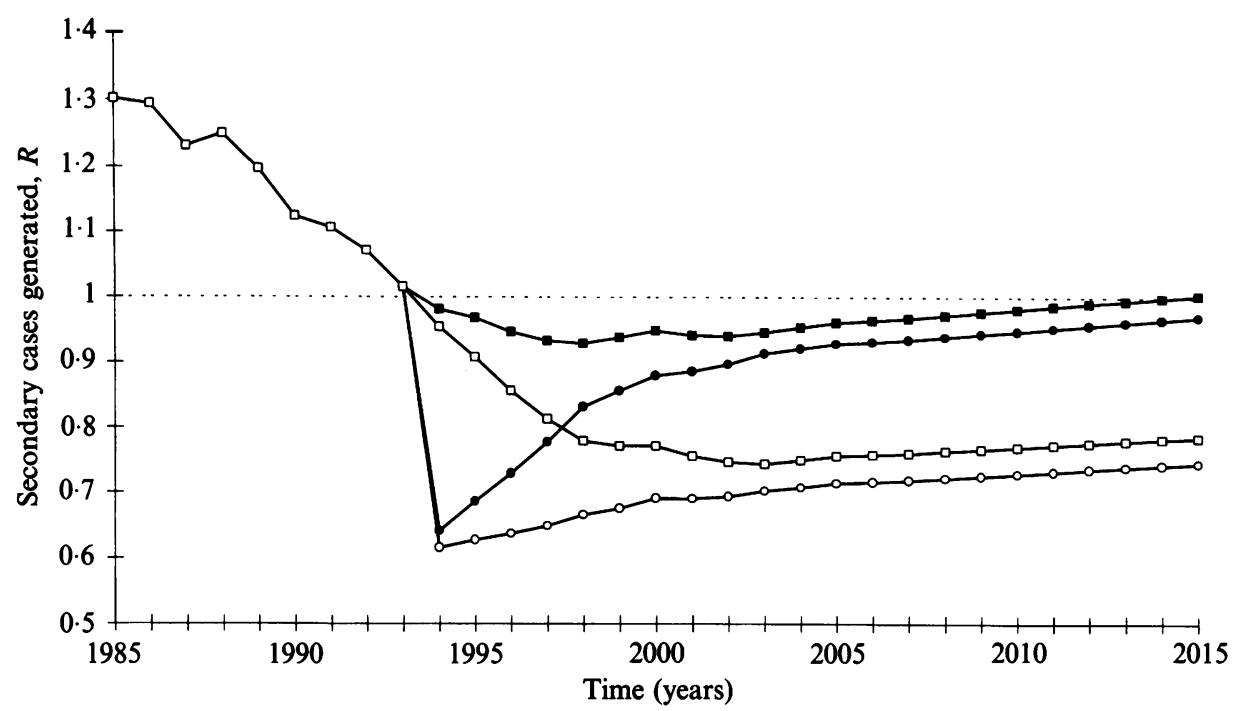

Fig. 12. Comparison between the predicted impact of a 2-dose policy with the second dose targeted at 4-year-olds, alone (squares), or introduced simultaneously with a school-age vaccination campaign (5-16-year-olds) (circles). In each case the difference between selective (open markers) and unselective (filled markers) administration of the second dose at age 4 is shown.

1 in 10 were vaccine failures. After the introduction of MMR and the elimination of infection, the total proportion susceptible rises to around $8 \%$ by the year 2000 , of which now 1 in 3 are vaccine failures. This assumes a vaccine efficacy of $90 \%$. Since all immunity will eventually be vaccine-induced, the long-term effect of $92 \%$ coverage with $90 \%$ efficacy will be almost equal numbers of susceptibles who are vaccine failures as those unvaccinated. Fig. $11 b, c$ illustrate the changes in the proportions susceptible under 2-dose selective or unselective, respectively. Targeting only those who have not previously received vaccine (Fig. 11b) ignores a large section of the susceptible population who are vaccine failures (shaded region); a group which will only be diminished by offering vaccine unselectively (Fig. 11c).

The predicted impact of introducing a 2-dose policy with the second dose at age 4 years simultaneous to a school-age child vaccination campaign in 1994 is shown in Fig. 12. This combination of policies brings about a rapid reduction in the potential for epidemics as the campaign reduces deficits in herd immunity, which is subsequently maintained if the 2-dose policy is applied unselectively.

Intermittent vaccination campaigns targeted at primary school children aged 5-11 years are recorded in Fig. 13. An interval of 5 years between pulses might be too long to prevent epidemics. A smaller interval might continually maintain $R<1$. Again the increased benefit of vaccinating irrespective of vaccination history (filled squares) is seen.

Fig. 14 provides some information on the timing of a campaign option. Since the introduction of MMR vaccination in 1988, the proportion of 5-10-year-olds who are susceptible is predicted to have declined as cohorts with higher-level vaccineinduced immunity age. This reaches a minimum in the mid-1990s and subsequently rises again, as a result, in part, of the elimination of infection which 


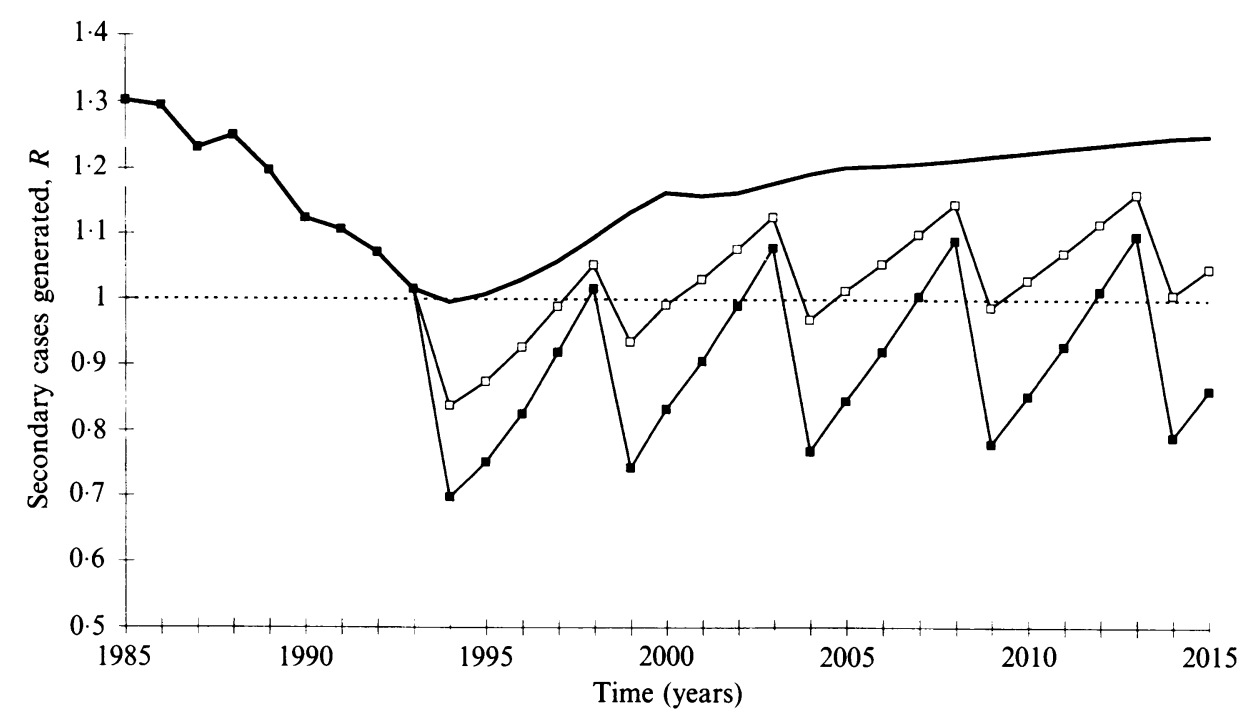

Fig. 13. The merits of a pulsed strategy, given selectively or unselectively, compared with the baseline policy. A pulse is administered every 5 years to primary school children (aged 5-11 years), either selectively (open marker), or unselectively (closed marker).

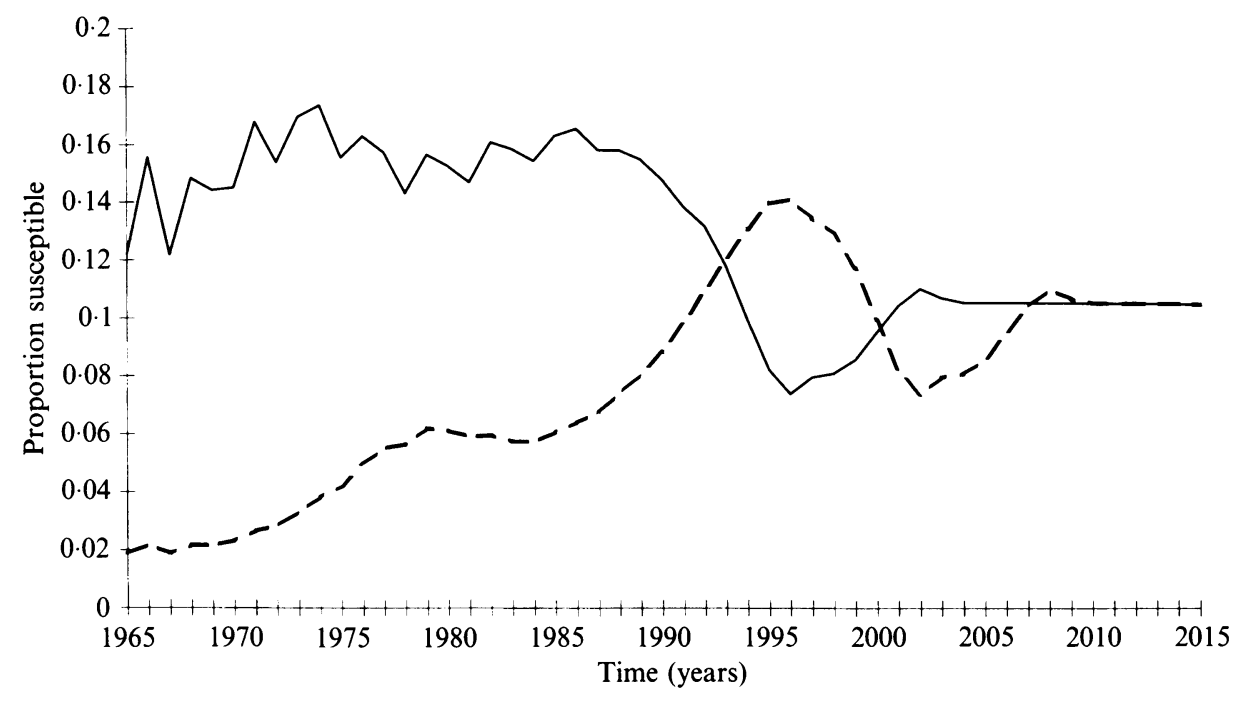

Fig. 14. Measles susceptibility in 5-10-year-olds (solid line) and 11-16-year-olds (dashed line) over the period 1965-2015.

no longer contributes to population immunity, and, in part, of a lower-level preschool vaccination in 4-5-year-olds [23]. Lagging some 5 years behind the 5-10 year group we see susceptibility first rise in the 11-16 year age group as infection is eliminated from the population (particularly from 1988 to 1993), and then decline to a minimum in the late $1990 \mathrm{~s}$ as the MMR-vaccinated cohorts move into this age class. Clearly the maximum susceptibility in the 11-16-year-olds is occurring now, as susceptibles move out of the 5-10 year age class. A delay in delivery of a campaign will enable these susceptibles to move beyond the school- 
age classes. These simulations of measles susceptibility in specific age classes are consistent with the trends in seroprevalence revealed by the PHLS countrywide surveys from 1986 to 1991 .

\section{DISCUSSION}

The analysis presented in this paper represents a detailed examination of the predictive capability of a mathematical model of the impact of mass vaccination on the epidemiology of an infectious disease. Whilst the same degree of precision in the model description of pre-vaccination dynamics is not sustained once vaccination has begun, it is clear that the trends described by a variety of outcome measures are certainly mirrored qualitatively, and much (although not all) of the quantitative detail is also captured.

Immediately following the introduction of mass vaccination model prediction significantly over-estimates observed measles incidence (scaled) and is out of synchrony with observed periodicity. One reason for this discrepancy could be that at the start of the vaccination programme measles vaccine was administered selectively to older children who had no history of infection [9], which would increase the estimate of the level of vaccine coverage. Further investigation is needed to confirm this conjecture. From 1988, following the introduction of MMR vaccine with ensuing increase in coverage of measles antigen, predicted cases mirror the observed trend towards negligible incidence early in the $1990 \mathrm{~s}$.

Over a period exceeding 2 decades, from 1969 to 1991, a good correlation is observed between predicted age-seroprevalence and serosurvey data. The most notable discrepancy is the under-estimation of seroprevalence in the 3-10-yearolds in 1986. A slight under-estimation is also apparent in earlier profiles for 1969 and 1979-83. Attempts to account for this difference as the result of changes in attendance to pre-school education since 1970 (which may have increased rates of infection in the under 5-year-olds), proved inconclusive.

The high degree of concordance between the observed age-distribution of notified cases and that predicted by the model, for the period up to 1986, is lost after the introduction of MMR vaccine. In 1991 the model significantly underestimates cases in children of less than 2 years of age. However, some of this discrepancy can be accounted for by age-related inaccuracy in clinical diagnosis recently identified by a PHLS study [15]. Whilst it is probable that there has always been a level of misdiagnosis in reported cases which is biased towards the youngest age classes, its importance will have increased with the decline in the circulation of measles accompanying high MMR vaccine coverage, and with the sporadic nature of measles infection.

In summary, it has not been possible fully to account for the discrepancies between the observed impact of vaccination on measles epidemiology in England and Wales and that predicted using the RAS model. The causes are likely to be multiple in origin, with misdiagnosis and inaccuracies in vaccine coverage statistics playing a prominent part. Additionally, at high-level vaccine coverage, as presently achieved in England and Wales, with resulting low incidence, it is probable that factors such as variation in vaccination coverage and in population density across the country, will have increasing influence over continued transmission. Furthermore, incidence becomes progressively less easy to capture 
using models which describe the average of transmission events which have become rare. Therefore, alternative models which incorporate elements of known spatial heterogeneity and have a stochastic basis may provide a useful framework for further studies into the persistence of childhood infections under strong vaccination pressure.

We believe that the assumption of $90 \%$ vaccine efficacy, which is at the lower end of reported estimates, is a sensible precaution in such analyses. Most estimates, determined from clinical diagnoses in outbreaks, would place vaccine efficacy nearer $95 \%$. Fine and Zell [25] suggest these may actually be biased towards under-estimation because the likelihood of outbreaks is higher where vaccine efficacy is lowest. However, clinical diagnosis may overlook individuals who acquire infection and become infectious to others, but who do not manifest symptoms. Thus, in our investigation where it is the transmission of infection rather than the occurrence of symptomatic infection which is of concern, vaccine efficacy estimates based on laboratory diagnosis have been chosen.

The 2 extremes of pattern of effective mixing in the community which gave quite similar results, provide some confidence in the robustness of the model. However, Gay and colleagues (personal communication) point out that the mixing parameters are determined from pre-vaccination data, where the vast majority of cases are in the first 10 years of life. This results in lower confidence in the estimates of values for older age classes, and it is these ages particularly secondary school and university age groups - that will be of increasing concern as the upward shift in the age distribution of infection continues.

Model projections suggest that the current policy of immunization may not be sufficient to eliminate measles from England and Wales in the long term at a level of vaccine efficacy of $90 \%$. A variety of amendments to current policy have been explored. A one-off campaign targeted at school-age children would reduce deficits in the herd immunity profile defined by serological surveys, and depress the potential for seasonal outbreaks, thus enhancing the effect of the current policy. Nevertheless, such a policy would not alter the predicted long-term outcome of a sustained potential for small outbreaks of infection. The optimal timing of a campaign is now, thus preventing highly susceptible cohorts moving beyond the school-age target group. For the long term the need is to prevent the build-up of susceptible numbers to epidemic proportions. Model predictions indicate the safest strategy for achieving such a goal is to administer a second dose of vaccine with the pre-school DT booster. Ideally, this would be administered irrespective of vaccination history, since it is predicted that vaccine failures will form a significant proportion of the susceptible population, and should be considered in combination with an initial school-age vaccination campaign.

We have not addressed the issue of vaccine compliance in this study. It has been assumed that vaccine can be administered equally effectively irrespective of method of delivery or target group. In practice we have little knowledge of exactly who would receive vaccine in a selective or an unselective policy. Circumstantial information is provided by compliance patterns for MMR vaccine and DT (diphtheria tetanus) pre-school booster vaccine. Data for the Welsh Districts, for example, in January 1994 (D. Thomas, personal communication), suggests that if the timing of a second dose of MMR vaccine coincided with the DT booster, a high 


\section{Measles vaccination in England and Wales}

coverage of $80 \%$ might result in a similar uptake level in those who had previously received a dose of MMR, but an uptake of less than 1/3 by those who had not previously received MMR vaccine. This suggests that in a 2-dose schedule it may be more easy to revaccinate vaccine-failures than to reach unvaccinated susceptibles.

\section{ACKNOWLEDGEMENTS}

This work was supported by a Department of Health contract (Grant No. 121/2782) and by the Wellcome Trust (grant support to R.M.A.). Our thanks go to Dr David Salisbury for numerous helpful discussions. D.J.N. is a Royal Society University Research Fellow.

\section{REFERENCES}

1. Department of Health. Immunisation against infectious disease. London: HMSO, 1988.

2. Miller E, Nokes DJ, Anderson RM. Measles, mumps and rubella vaccination. BMJ 1992; 304: 1440-1.

3. Office of Population Censuses and Surveys. Statistics of infectious diseases. London: HMSO, 1974-1993.

4. Carter H, Gorman D. Measles, mumps, and rubella vaccine: time for a two stage policy? BMJ 1992; 304: 779.

5. Registrar General's Statistical Review. Annual review of the Registrar General of England and Wales. London: HMSO, 1948-1973.

6. Nokes DJ, Anderson RM. The use of mathematical models in the epidemiological study of infectious diseases and in the design of mass immunization programmes. Epidemiol Infect 1988: 101 : 1-20.

7. Hamer W. Epidemic disease in England. Lancet 1906; i : 733-9.

8. Fine PEM, Clarkson JA. Measles in England and Wales. I. An analysis of factors underlying seasonal patterns. Int J Epidemiol 1982; 11 : 5-14.

9. Schenzle D. An age-structured model of pre- and post-vaccination measles transmission. IMAJ Math Appl Med Biol 1984; 1 : 169-91.

10. Anderson RM, May RM. Age-related changes in the rate of disease transmission: implications for the design of vaccination programmes. J Hygiene 1985; 94 : 365-436.

11. Bolker BM, Grenfell BT. Chaos and biological complexity in measles dynamics. Philosoph Trans R Soc Lond B Biol Sciences $1993 ; 251$ : 75-81.

12. Anderson RM, Nokes DJ. Mathematical models of transmission and control. In: Holland WW, Detels T, Knox G, eds. Oxford textbook of public health. Oxford: Oxford Medical Publications, 1991.

13. Ukkonen $\mathrm{P}$, Von Bonsdorf C-H. Rubella immunity and morbidity: effects of vaccination in Finland. Scand J Infect Dis 1988; $20: 255-9$.

14. Grenfell BT, Anderson RM. The estimation of age-related rates of infection from case notifications and serological data. J Hygiene $1985 ; 95$ : 419-36.

15. Brown D, Ramsay M, Richards A, Miller E. Salivary diagnosis of measles: a study of notified cases in the United Kingdom, 1992-93. BMJ 1994; 308: 1015-17.

16. Morgan-Capner P, Wright J, Miller CEM. Surveillance of measles, mumps and rubella by age. BMJ 1988; 297 : 770-2.

17. Farrington CP. Modelling forces of infection for measles, mumps and rubella. Stat Med $1990 ; 9: 953-67$.

18. Anderson RM, Grenfell BT. Quantitative investigations of different vaccination policies for the control of congenital rubella syndrome (CRS) in the United Kingdom. J Hyg 1986; 96 : $305-33$.

19. Anderson RM, May RM. Vaccination against rubella and measles: quantitative investigations of different policies. J Hyg 1983; 90 : 259-325.

20. Anderson RM, May RM. Infectious diseases of humans: dynamics and control. Oxford: Oxford University Press, 1991. 
21. Department of Education and Science. Statistical Bulletin. Government Statistical Service, 1982-1991.

22. Department for Education. Statistical Bulletin. Government Statistical Service, 1992.

23. Department of Health Statistics Division. Vaccination and immunisation, England. Government Statistical Service, 1968-1993.

24. Office of Population Censuses and Surveys. Birth Statisties, 1968-1993. OPCS No. 13 and 20. London: HMSO.

25. Fine PEM, Zell ER. Outbreaks in highly vaccinated populations - implications for studies of vaccine performance. Amer J Epidemiol 1994; 139: 77-90.

26. Morse D, O'Shea M, Hamilton G, et al. Outbreak of measles in a teenage school population: the need to immunize susceptible adolescents. Epidemiol Infect 1994; 113 : 355-65.

27. Calvert N, Cutts F, Miller E, Brown D, Munro J. Measles among secondary school children in west Cumbria: implications for vaccine policy. CDR Review 1994; 4: R70-6.

28. Walker J, Nokes DJ, Jennings R. Longitudinal study of toxoplasma in South Yorkshire. Epidemiol Infect 1992; 108: 99-106.

29. Cox MJ. Seroepidemiology of measles, mumps and rubella in St. Lucia and Jamaica [thesis]. London: London University, 1989.

30. Begg N, White J, Handford S. "COVER" (Cover of vaccination evaluated rapidly) 13. Commun Dis Rep 1990; 90(13): 3-4.

31. Department of Health. Immunisation against infectious disease. London: HMSO, 1993.

32. Agur Z, Cojocaru L, Mazor G, Anderson RM, Danon YL. Pulse mass measles vaccination across age cohorts. Proc Nat Acad Sci USA 1993; 90 : 11698-702.

33. Bailey NJT. On estimating the latent and infectious periods of measles. 1. Families with two susceptibles only. Biometrika $1956 ; 43$ : 15.

34. Bailey NJT. The mathematical theory of infectious diseases. London: Griffin, 1975.

35. Nokes DJ, Wright J, Morgan-Capner P, Anderson RM. Serological study of the epidemiology of mumps virus infection in North-West England. Epidemiol Infect 1990; 105: 175-95. 